\title{
FOXM1 in sarcoma: role in cell cycle, pluripotency genes and stem cell pathways
}

\author{
Fergal C. Kelleher ${ }^{1,2}$ and Hazel O'Sullivan ${ }^{3}$ \\ 1 St. James Hospital, Dublin, Ireland \\ 2 Trinity College Dublin, Dublin, Ireland \\ ${ }^{3}$ Whangarei Base Hospital, Whangarei, New Zealand \\ Correspondence to: Fergal C. Kelleher, email: fergalkelleher@hotmail.com \\ Keywords: FOXM1, pluripotency genes, thiazole antibiotics, DREAM, mesenchyme \\ Received: November 27, 2015 Accepted: March 29, 2016 \\ Published:April 09, 2016
}

\section{ABSTRACT}

FOXM1 is a pro-proliferative transcription factor that promotes cell cycle progression at the G1-S, and G2-M transitions. It is activated by phosphorylation usually mediated by successive cyclin - cyclin dependent kinase complexes, and is highly expressed in sarcoma. p53 down regulates FOXM1 and FOXM1 inhibition is also partly dependent on Rb and p21. Abnormalities of p53 or Rb are frequent in sporadic sarcomas with bone or soft tissue sarcoma, accounting for $36 \%$ of index cancers in the high penetrance TP53 germline disorder, Li-Fraumeni syndrome.

FOXM1 stimulates transcription of pluripotency related genes including SOX2, KLF4, OCT4, and NANOG many of which are important in sarcoma, a disorder of mesenchymal stem cell/ partially committed progenitor cells. In a selected specific, SOX2 is uniformly expressed in synovial sarcoma. Embryonic pathways preferentially used in stem cell such as Hippo, Hedgehog, and Wnt dominate in FOXM1 stoichiometry to alter rates of FOXM1 production or degradation. In undifferentiated pleomorphic sarcoma, liposarcoma, and fibrosarcoma, dysregulation of the Hippo pathway increases expression of the effector co-transcriptional activator Yes-Associated Protein (YAP). A complex involving YAP and the transcription factor TEAD elevates FOXM1 in these sarcoma subtypes. In another scenario $80 \%$ of desmoid tumors have nuclear localization of $\beta$-catenin, the Wnt pathway effector molecule. Thiazole antibiotics inhibit FOXM1 and because they have an auto-regulator loop FOXM1 expression is also inhibited. Current systemic treatment of sarcoma is of limited efficacy and inhibiting FOXM1 represents a potential new strategy.

\section{INTRODUCTION}

Soft tissue sarcomas are heterogeneous mesenchymal tumors. The American Cancer Society in 2015 documented an incidence rate of 11,930, with an anticipated annual death rate of 4,870. Treatment of metastatic disease usually comprises doxorubicin and ifosfamide either alone or in combination, with a greater than $40 \%$ rate of 12 - week progression free survival considered a descriptor of second-line treatment efficacy. Therefore the benefit from systemic treatment of this disease is modest with a need for new therapeutics. One candidate strategy is targeted inhibition of the mammalian transcription factor Forkhead Box M1 (FOXM1).

\section{FOXM1 AND THE CELL CYCLE}

FOXM1 is a member of the Forkhead transcription factor family. Constituent members have an evolutionarily conserved, 100-amino acid long DNA binding domain called Forkhead or 'winged helix' [1, 2]. In Drosophila melanogaster mutations of forkhead create ectopic head structures in the fruit fly embryos, hence the nomenclature. There are 19 different subgroups, FOX1-FOXS, categorized on the basis of sequence homology inside and outside the forkhead domain. In particular FOXA, FOXC, FOXM, FOX0 and FOXP are essential components of oncogenic and tumor suppressive pathways. FOXM1 is a crucial pro-proliferative transcription factor, which is activated by phosphorylation. It also has an upregulating auto regulatory loop [3]. It is induced by oncoproteins such 
as MYC and KRAS and repressed by products of tumor suppressor genes such as CHK2 and TP53 [4-6]. FOXM1 transcriptionally activates important pro-proliferative genes and promotes cell cycle progression at the G1/S and G2/M transitions. The cyclin-dependent kinases CDK4/6 phosphorylate FOXM1 to facilitate continued expression of G1/S phase genes [7]. FOXM1 undergoes cytoplasmic accumulation in late $\mathrm{G} 1$ and $\mathrm{S}$ phases, followed by cyclin E-CDK2 / Raf-MEK-ERK mediated phosphorylation, nuclear translocation and entry into G2-M phase $[8,9]$. In normal cells, FOXM1 is phosphorylated in the S to G2 phases, and undergoes ubiquitin dependent proteasomal destruction during the M to G1 phase.

Cyclin/CDK complexes mediate cell cycle progression with their effects partly executed by altering transcription factors such as FOXM1 or E2F. The E2F1 transcription factor also contributes to the expression of FOXM1 [1]. Cyclins markedly activate the catalytic activity of their serine/threonine cyclin dependent kinase partner with activity of FOXM1 mediated by successive phosphorylation events. RB is also an important substrate for cyclin-CDK complexes. Early in the cell cycle at M/G1 transition almost all of the phosphate groups are removed from retinoblastoma protein $(\mathrm{pRb})$ resulting in an unphosphorylated configuration. With progression through the G1 phase a single phosphate group is attached to any of 14 potential phosphorylation sites. At the restriction point in late $\mathrm{G} 1$ phase, $\mathrm{pRb}$ is phosphorylated by cyclin E- CDK2 complexes at a minimum of 12 more sites creating a hyperphosphorylated state, which persists until entry into the $\mathrm{M}$ phase. The active form of $\mathrm{RB}$ is the unphosphorylated protein, which binds cellular proteins including E2F. E2F family transcription factors are required for expression of S-phase genes. When $\mathrm{pRb}$ is hyperphosphorylated this causes the release of transcription factors including E2F permitting G1 to $\mathrm{S}$ phase transitions and cell cycle progression $[10,11]$. Importantly two potential E2F binding sites have been identified in the FOXM1 promoter [1]. The FOXM1 promoter also binds B-Myb and CHR-NF-Y. Children with hereditary retinoblastoma, a condition in which tumors arise from biallelic functional loss of $R B 1$, have a 1,000 fold increased risk of osteosarcoma compared to the general population. Furthermore $R B 1$ alterations are identified in $80 \%$ of primary sporadic osteosarcomas [1214]. Amplification of $C D K 4$ and loss of $R B 1$, or $C D K N 2 A$ loss are considered nearly universal in osteosarcoma with $20 \%$ of cases having either amplification of $C D K 4$ or deletion of $C D K N 2 A$ [15]. These alterations lead to $\mathrm{G} 1 / \mathrm{S}$ deregulation.

Growth factors neutralize the inhibitory effects of $\mathrm{Rb}$ by its successive phosphorylation. The G1/S checkpoint is the first important checkpoint in the cell cycle and it involves both the RB and p53 proteins. RB and p53 have been implicated in numerous sarcoma subtypes. Mice with osteoblast-restricted deletion of p53 and pRb develop short latency high-grade osteosarcoma [11]. In childhood survivors of retinoblastoma, osteosarcoma is the most common subsequent malignancy to arise, itself a disease stemming from homozygous functional loss of $R B$. In this population osteosarcoma can originate either in irradiated or non-irradiated tissue and represents $25 \%-40 \%$ of second cancers [16, 17]. The DREAM (Dimerization partner, RB-like, E2F and multi-vulval class B (MuvB)) complex mediates gene repression in G0, early and late phases of the cell cycle and co-ordinates periodic gene expression. Peaks in gene expression occur in G1/S and G2/M [18]. $\mathrm{E} 2 \mathrm{~F}$ is required for $\mathrm{G} 1 / \mathrm{S}$ phase gene expression. MuvB core complex, BMYB and FOXM1 are required for G2/M phase gene expression. BMYB (a transcription factor phosphoprotein) and FOXM1 are phosphorylated and undergo proteasomal destruction during respective $S$ to $\mathrm{G} 2$, and $\mathrm{M}$ to $\mathrm{G} 1$ phases. In cancer increased BMYBMuvB-FOXM1 activity supervenes with loss of DREAM function with a shift in cellular balance from quiescence to proliferation.

The p53 transcription factor is a tumor suppressor protein, which down-regulates FoxM1. TP53 is mutated in numerous cancers including many sarcoma subtypes. The highly penetrant cancer predisposition disorder LiFraumeni syndrome is associated with germline TP53 mutations. The index cancers in $36 \%$ of patients with LiFraumeni syndrome are either bone or soft tissue sarcomas [19]. TP53 mutations occur in $19 \%$ to $38 \%$ of sporadic osteosarcomas [20, 21].

FOXM1 is itself a transcription factor that regulates numerous $\mathrm{G} 2 / \mathrm{M}$ specific genes. Inhibition of FOXM1 is also partly dependent on $\mathrm{Rb}$ and $\mathrm{p} 21$. Secondary to DNA damage, p53 can participate in repressing FoxM1 mRNA and thus FOXM1 protein expression. A global gene expression analysis of different cancer types found FOXM1, E2F1, and MYBL2 are disproportionately upregulated in p53 mutant cancers [22]. MYB-related protein B (B-MYB/MYBL2) coordinates cell cycle transition in conjunction with multivulval class B (MuvB) complex and FOXM1[23]. The binding of FoxM1 to the promoters of genes involved in $\mathrm{G} 2 / \mathrm{M}$ is dependent on the transcription factor B-Myb [24]. The transcriptional activation of FoxM1 requires sequential phosphorylation by cyclin dependent kinases and PLK1. These are target genes of B-Myb. Therefore B-Myb enables FoxM1 binding to $\mathrm{G} 2 / \mathrm{M}$ gene promoters. Microarray analysis of breast cancers demonstrates that FOXM1 regulates genes essential for chromosomal segregation and mitosis with loss of FOXM1 leading to mitotic spindle defects and mitotic catastrophe [25]. FOXM1 is progressively phosphorylated with initiation of phosphorylation in mitosis as demonstrated in Figure 1, and is involved in G2-M phases of the cell cycle. Expression of FOXM1 is increased during G1 and $\mathrm{S}$ phases and is maximal in G2-M phases of the cell cycle with overexpression of FOXM1 stimulating expression of cyclin B1[26]. 


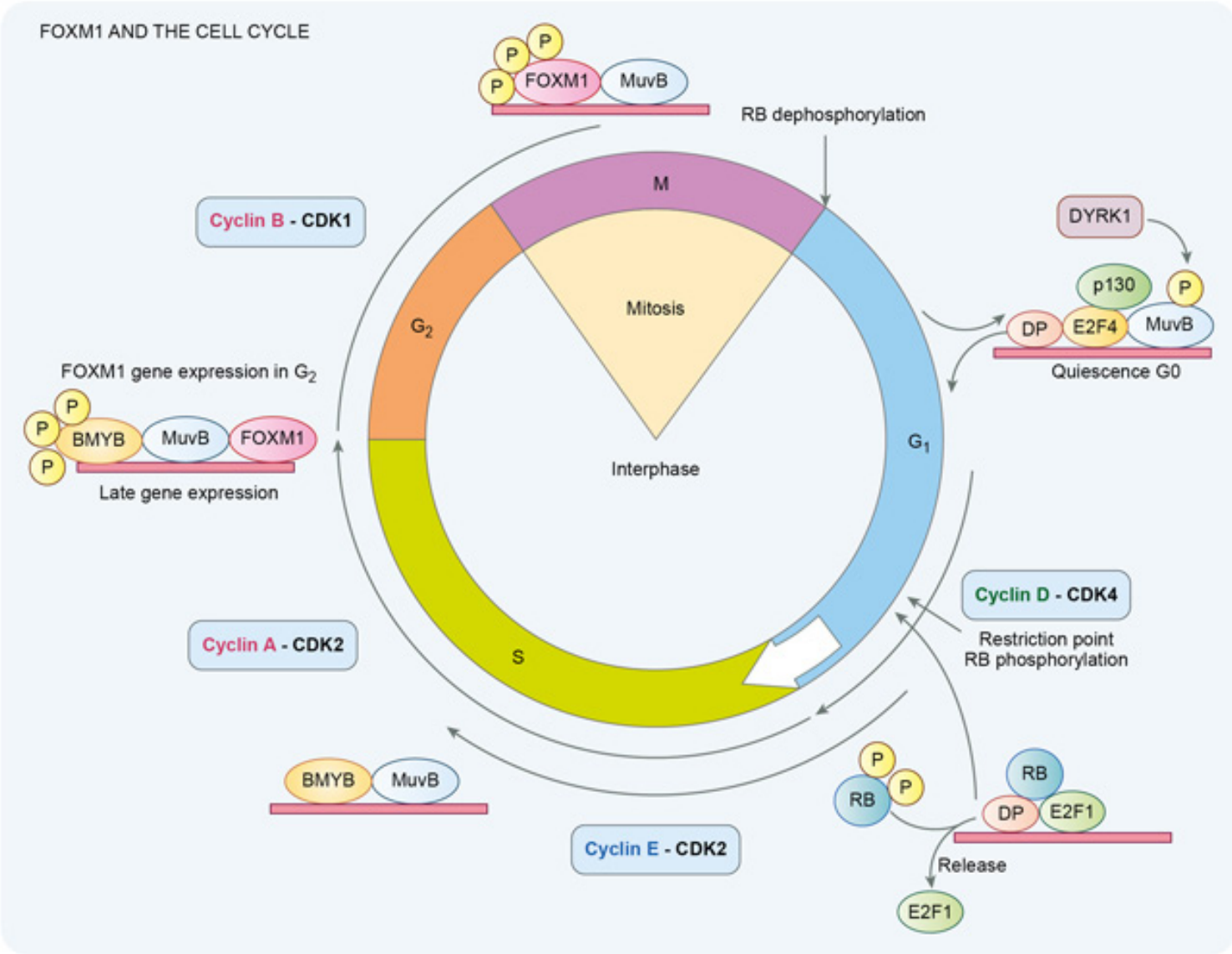

FLUCTUATING LEVELS OF CYCLINS DURING CELL CYCLE
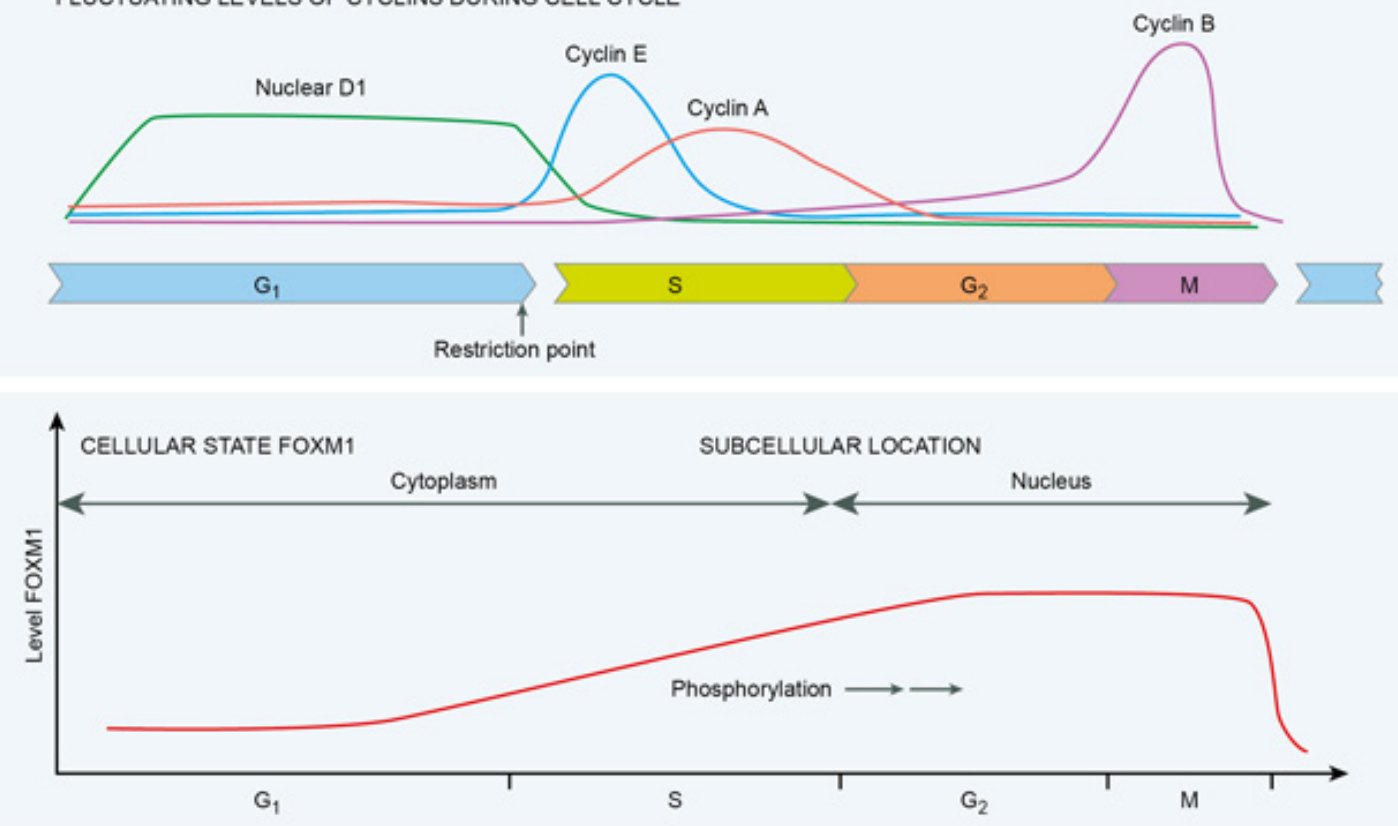

Figure 1: Temporal dynamics of FOXM1 in the cell cycle 


\section{FOXM1 AND MESENCHYMAL TUMORS}

FOXM1 has pleiotropic roles in embryogenesis in which it is almost ubiquitously expressed, particularly in proliferating mesenchymal and epithelial cells. In adult tissues expression is restricted to actively dividing cells of selected organs with expression eliminated in quiescent or terminally differentiated cells [2]. Interestingly the $\mathrm{Rb}$ pathway controls both quiescence and senescence. A senescence - quiescence switch mediated by cross talk between the Rb-AKT signaling pathways, FoxO3a and FoxM1 transcription factors has been identified in cultured fibroblasts [27]. Senescence is an important barrier to cancer, which is overcome with development of malignancy. Furthermore in these studies E2F1 and E2F3 were found to bind to and activate the gene promoter of FOXM1 in proliferating fibroblasts. In senescent cells, CDK4 and CDK6, targets of p16 $6^{\mathrm{INK} 4 \mathrm{a}}$ stabilize FOXM1 protein [7]. A systematic screen of CDK4/6 substrates identified phosphorylation of FOXM1 as a common target [7]. CDK4/6 stabilized and activated FOXM1, thereby maintaining G1/S phase gene expression and suppressing reactive oxygen species levels. Phosphorylation of FOXM1 suppressed senescence in malignant cells. In malignant peripheral nerve sheath tumors, amplification of the $C D K 4$ gene and increased levels of FOXM1 protein are significant independent predictors of poor survival [28].

The World Health Organization has a tissue of origin classification system for sarcomas with subtype assignment determined by histiogenesis. Limitation arises as it is not possible to assign a lineage of origin to some poorly differentiated tumors. Interestingly in two malignancies separate from sarcoma, embryonic carcinoma and neuroblastoma, a role for FOXM1 has been identified in the maintenance of the undifferentiated tumor state of the malignant cells. This arises by FOXM1 activating pluripotency-associated genes $[29,30]$. Sarcoma likely arises from stem cells or partly committed progenitor cells. A suggested inference is that FOXM1 has a similar role in undifferentiated pleomorphic sarcoma. It is a diagnosis of exclusion as these tumors defy lineage assignment. This postulate is partly substantiated by recent experimental evidence in which deregulated Hippo pathway signaling in soft-tissue sarcomas was found to promote FOXM1 expression and tumorigenesis in undifferentiated pleomorphic sarcoma, fibrosarcoma and liposarcoma [31]. Most soft-tissue sarcomas arise from tissues derived from the mesodermal or ectodermal germ layers, however some such as angiosarcomas have an endodermal origin. Therefore the ontological merit in evaluating FOXM1 as a therapeutic target in sarcoma, especially undifferentiated pleomorphic sarcoma, is apparent. Furthermore FOXM1 participates in epithelial to mesenchymal transition, a determinant of the morphological subtypes of synovial sarcoma.
FOXM1 levels can be increased by gene amplification and translocation, or by altered kinetics of production and degradation. FOXM1 maps to chromosomal band 12p13 [32]. This locus is amplified in several types of cancer with FOXM1 amplification among the most prevalent molecular aberration in nonHodgkin's lymphoma [33]. Chromosome band 12q13 is amplified in malignant peripheral nerve sheath tumors [34]. Comparative genomic hybridization of these tumors has permitted correlation of regional copy number gains with decreased survival $[35,36]$. FOXM1 emerged as one candidate amplified gene, however on multivariate analysis $C D K 4$, also on chromosome $12 \mathrm{q} 13$, was the most significant forecaster of poor survival [28]. Amplification of chromosomal region 12q13-15 is also found in welldifferentiated liposarcomas within supernumerary ring and giant marker chromosomes. The proto-oncogenes $C D K 4$ and $M D M 2$ are both amplified in well-differentiated liposarcoma. Translocations involving chromosome band12q13 occur in other soft tissue sarcomas subtypes. Myxoid/round cell liposarcoma has a translocation $\mathrm{t}$ $(12 \mathrm{q} 13 ; 16 \mathrm{p} 11)$ that creates a FUS-DDIT3 gene fusion. Clear cell sarcoma has a translocation $\mathrm{t}(12 \mathrm{q} 13 ; 22 \mathrm{q} 12)$ that creates an EWSR1-WT1 gene fusion. Infantile fibrosarcoma has a translocation $\mathrm{t}(12 \mathrm{q} 13 ; 15 \mathrm{q} 26)$ that creates an ETV6-NTRK3 gene fusion. The frequency of the described fusion genes in the respective malignancies is each $\sim 75 \%$. Considering a phylogenetic map of soft tissue sarcomas (which comprises $\sim 65$ histological types), Ewing sarcoma family tumors and clear cell sarcoma occupy a common second order phylogenetic branch. FOXM1 is of established importance in both these malignancies. Welldifferentiated liposarcomas and myxoid liposarcomas arise from a common first order branch but are separate from other FOXM1 candidate soft tissue sarcomas.

Direct binding to the FOXM1 promoter by E2F or c-Myc increases its expression by up regulating gene transcription. Conversely, p53 negatively regulates expression of FOXM1[37]. Mutation or inactivation of p53 frequently occurs in malignancies and may increase FOXM1 expression. Mice with p53 deficiency develop spontaneous sarcomas and thymic lymphoma [38, 39]. Li-Fraumeni syndrome, which arises from germline mutations in TP53, is informative. Affected individuals are at markedly increased risk of developing the following six core malignancies: CNS tumors, breast cancer, acute leukemia, adrenocortical carcinoma, soft tissue and bone sarcomas. [40]. Loss of function alterations in p53 typically leads to resistance to apoptosis because p53 stimulates pro-apoptotic gene expression including Noxa, Bad, Bax, DR4, Puma, Apaf1 and caspase-6 [41]. P53 loss is a frequent molecular aberration in sporadic sarcoma.

The FOXM1c isoform binds to and transactivates the E-cadherin gene promoter at a conserved FOXM1 binding site in mice and humans [42]. E-cadherin is a trans-membrane glycoprotein with tumor suppressor 
activity that participates in intercellular adhesion at adherens junctions. Down regulation of E-cadherin is one part of the phenomenon of epithelial-mesenchymal transition (EMT) in which cells lose apical-basal polarity and mesenchymal genes are induced. EMT has diverse biological roles in gastrulation, tumor invasion and metastasis. In synovial sarcoma, a monophasic fibrous variant accounts for $\sim 60 \%$ of cases with the remainder being biphasic synovial sarcoma (containing epithelial and spindle cell components), a rare monophasic epithelial type, and a poorly differentiated type. EMT accounts for this variation in histological appearance. The idea that FOXM1 transcriptionally upregulates a tumor suppressor gene is unexpected and a context dependent approach to FOXM1's role in malignancy must be inferred. FOXM1 would appear to be influential in biphasic synovial sarcomagenesis. Contrastingly in non-small cell lung cancer, high expression of FOXM1 is correlated with loss of E-cadherin expression and anomalous immunopositivity for vimentin [43]. FOXM1 expression is associated with lymph node metastasis and is also an independent adverse predictor of outcome in this variety of lung cancer.

Finally, FOXM1 is important in rhabdomyosarcoma wherein it directly binds to the Bublb promoter, which has a FOXM1 consensus-binding site [44]. Bub1b is a spindle assembly checkpoint protein that is of critical importance for growth and survival of both alveolar and embryonic rhabdomyosarcoma cells. There is excellent correlation between FOXM1 and Bub1b levels in this disease. A FoxM1/Bub1b signaling pathway is an identified required component for survival and growth of rhabdomyosarcoma [45]. Bub1b knockdown suppresses in-vivo tumor growth with regression of established tumors affected by postmitotic endoreduplication checkpoint mitotic catastrophe. Using a chromatin immunoprecipitation assay Bublb has been established as a direct transcriptional target of FoxM1. Experimental suppression of FOXM1 transcription using the FOXM1 inhibitor Siomycin (an antibiotic thiazole compound) or shRNA reduced the expression of Bub1b and inhibited cell growth and survival. Interestingly, patients with mosaic variegated aneuploidy syndrome, a rare disorder with has biallelic or heterozygous mutations of the $B u b 1 B$ gene, have constitutional aneuploidy and a predisposition to early childhood cancer including rhabdomyosarcoma, leukemia and Wilm's tumor $[44,46]$. Targeting FOXM1 may prove a useful future therapeutic strategy in rhabdomyosarcoma.

\section{FOXM1 AND EXPRESSION OF PLURIPOTENCY GENE}

FOXM1 stimulates transcription of pluripotencyrelated genes in cancer stem cells, which are cells with properties of multi-lineage differentiation and selfrenewal capacity. Experimental evidence substantiating this concept, detailed herein, has been described in neuroblastoma, Ewing sarcoma, embryonal carcinoma and synovial sarcoma. These malignancies are diagnosed in childhood, adolescence and young adults, respectively. Thus, there is a recurring theme of temporal correlation between tumors arising in childhood or early adult life with a pathobiology of upregulated pluripotency genes. Sarcomas of bones are more common in adolescents and young adults. Though as a categorical group soft tissue sarcomas are more common with increasing age many exceptions apply, such as rhabdomyosarcoma- median age at diagnosis 5 years, and synovial sarcoma occurring most often in young adults- average age at diagnosis 35 years [47]. The median age at diagnosis of translocationassociated sarcomas (e.g. desmoplastic small round cell, Ewing, clear cell, synovial, myxoid-round cell liposarcoma) is 20-50 years, and 50-70 years in complex types of sarcoma (e.g. malignant peripheral nerve sheath tumor, fibrosarcoma, leiomyosarcoma, undifferentiated pleomorphic sarcoma).

In neuroblastoma FoxM1 activates SOX2 (Sex determining Y-box 2) expression [48]. SOX2 along with K1f4, Oct4 and c-Myc is one of the four 'Yamanaka' transcription factors that reprogram differentiated cells into induced pluripotent stem cells. In Ewing sarcoma an EWS-FLI1 gene translocation is identified in $\sim 85 \%$ of cases. This chimeric fusion gene encodes an aberrant transcription factor and is correlated with increased in vitro cell line levels of FOXM1 [49]. Raised FOXM1 levels are also expressed in human Ewing sarcoma tumors. There is an established relationship between EWS-FLI1 and the pluripotency genes SOX2,OCT4, and NANOG in Ewing sarcoma. EWS-FLI-1 represses the miRNA145 promoter and induces expression of $S O X 2$ in Ewing sarcoma to initiate the reprogramming of mesenchymal stem cells towards cancer stem cells [50]. In Ewing sarcoma SOX2 enables cell proliferation and survival by regulating p27, p21 and cyclin $E$ to facilitate $\mathrm{G} 1 / \mathrm{S}$ phase transition. In a systematic evaluation of Ewing sarcoma cell lines, human samples and xenograft models siRNA mediated inhibition of SOX2 induces apoptosis and G1/S phase arrest [51]. Ewing sarcoma expresses neurogenic genes, and the postulated cell of origin of Ewing sarcoma is either a mesenchymal stem cell or primitive early neuro-ectodermal cell. EWS-FLI-1 also induces the expression of SOX2, OCT4 and NANOG in human pediatric mesenchymal stem cells but not in the adult counterparts. CD133 is a stem cell marker in Ewing sarcoma and can be used to identify subpopulations of cells with tumor initiating activity as well as the ability to sustain growth when serially xenotransplanted [52]. CD133 expressing Ewing sarcoma cells are capable of chondrogenic, osteogenic and adipocytic differentiation. CD133 expressing cells expressed higher levels of NANOG, SOX2 and OCT4 [53]. Cancer stem cells in Ewing sarcoma are involved in chemo resistance, with 
in vitro musculoskeletal sarcoma stem cells expressing SOX2, OCT3/4 and NANOG $[54,55]$.

SOX2 is uniformly expressed in synovial sarcoma and its expression is an essential requirement for proliferation of these cells. SOX2 occupancy of the chromatin remodeling complex BAF (SWI/SNF) is correlated with decreased histone $\mathrm{H} 3$ lysine 27 trimethylation (H3K27me3) an epigenetic phenomenon. The characteristic t (X: 18)(p11.2; q11.2) translocation of synovial sarcoma fuses the SS18 gene on chromosome 18 to one of three homologous genes, SSX1, SSX2 or SSX4, which cluster on the X chromosome [56]. SS18 is an integral subunit of the BAF complex and is closely associated with the catalytic Brg subunit. In synovial sarcoma SS18-SSX replaces SSX18 in the BAF complex and evicts the tumor suppressor BAF47 that then undergoes proteasomal degradation [57]. This altered BAF complex binds with the SOX2 locus and reverses polycomb mediated gene repression thereby activating Sox2. A 2-amino acid segment is the decisive discriminator for SNF5 ejection and SOX2 induction [58] i.e. the non-pathogenic partner gene SSX3 harbors a methionine-isoleucine amino-acid pair, rather than the evicting lysine-arginine amino acid doublet of SSX1, SSX2, and SSX4 within the chimeric SS18: SSX gene fusion. A future therapeutic approach has been postulated using a decoy molecule that causes the evicting amino acid doublet of the expressed SYT: SSX fusion gene to resemble the innocent SSX3 protein. The role of FOXM1 in synovial sarcoma needs experimental evaluation. However, inferring its effect on upregulating pluripotency genes and in particular activation of SOX2 in other tumors raises an important question: Does inhibition of FOXM1 vicariously targets SOX2 in synovial sarcoma? This postulate merits future research.

SOX2 also maintains the self-renewal of osteosarcoma tumor initiating cells [56]. In this malignancy SOX2 opposes the cellular differentiating effect of the Wnt pathway, which in turn can block SOX2 expression [59]. Interestingly constitutive Wnt/ $\beta$ catenin signaling is aberrantly activated by SYT-SSX2 in synovial sarcoma [60]. In a general perspective SOX2 has been found to antagonize the Hippo pathway to maintain 'stemness' in cancer cells [58]. Evidence also exists that Yes-Associated Protein (YAP), the effector molecule which is inhibited by the Hippo signaling pathway cascade of cytoplasmic kinases, is increased in a subset of soft tissue sarcoma [31]. In these sarcomas i.e. fibrosarcoma, undifferentiated pleomorphic sarcoma and liposarcoma, YAP dependent expression of FOXM1 is necessary for cell proliferation and tumorigenesis.

Embryonic stem cells, embryonic germ cell and embryonal carcinoma (EC) cells are pluripotential cells. FOXM1 is required to maintain embryonal carcinoma cell pluripotency [30]. Embryonal carcinoma cells are derived from teratocarcinomas, with mice P19 EC cells derived from a genetically engineered murine teratocarcinoma. These cells can be induced to differentiate in vitro to any cell type of all three germ layers. There is an aforedescribed interesting parallel as sarcomas usually derive from mesodermal or ectodermal germ layers, in contrast to carcinomas, which arise from the endodermal germ layer. Some sarcomas such as angiosarcomas however have an endodermal origin establishing that soft tissue sarcomas as a group of heterogenous diseases with a possible common phylogenetic origin can arise from or developmentally transit all three germ layers. FOXM1 binds to and stimulates the Oct4 prompter in P19 EC cells. FOXM1 expression is repressed during retinoic acid induced differentiation of early stage P19 EC cells and FOXM1 knockdown in EC cells causes spontaneous differentiation to mesodermal derivatives such as adipose tissue or muscle [30]. Maintaining FOXM1 expression prevents down regulation of Nanog and Oct4 during P19 differentiation. When FOXM1 is overexpressed in human newborn fibroblasts or retinoic-acid differentiated cells, expression of Nanog, Sox 2 and Oct4 is reinstated. Therefore the FOXM1 transcription factor maintains the pluripotency of P19 EC cells by up regulating pluripotency genes in this malignancy. Retinoic acid induced differentiation of human NT2/D1 embryonic carcinoma cells decreases expression of FOXM1 [61]. In a preclinical investigation this was found to be mediated by increased levels of miR-134 which binds to the FOXM1 3'UTR. In general miRNAs negatively regulate gene expression and are loaded onto RNA induced silencing complexes (RISC) with the miRNAs interacting with targeted messenger RNA. This interaction involves imperfect base pairing with microRNA response elements usually identified within the 3'UTR of the messenger RNA.

The general importance of FOXM1 upregulating pluripotency genes such as $S O X 2, O C T 4$, and $N A N O G$ is apparent beyond the specific cited examples. The histology of many sarcomas suggests a cellular pluripotency model of origin. Firstly, the World Health Organization (WHO) has a lineage of origin classification but some tumors such as hemangiopericytoma, fibrous histiocytoma or phosphaturic mesenchymal tumor do not have an identifiable lineage of origin. Sarcomas, which are poorly differentiated, are also increasingly difficult to assign a lineage of origin. The most common soft tissue sarcoma is undifferentiated pleomorphic sarcoma, a diagnosis of exclusion. Some sarcomas have histologic juxtaposition of malignant cells of different germ layer origin such as biphasic synovial sarcoma having both glandular cells (endodermal origin) and spindle cells (mesenchymal origin). Tumors with heterologous rhabdomyoblastic components also have close apposition of cells with different origins. This latter group has a sub-category of tumors with sarcomatous elements only (malignant mesenchymoma, dedifferentiated chondrosarcoma and dedifferentiated liposarcoma). Other subcategories with 
heterologous rhabdomyoblastic components include tumors with epithelial components (e.g. carcinosarcoma), malignant mixed Mullerian tumors, tumors with sex cord or stromal elements, and tumors of neuroectodermal derivation (e.g. medulloblastoma). A further observation supporting the pluripotent cell of origin in sarcomagenesis is the spatial heterogeneity of sarcoma types, often identified in body parts lacking a normal tissue of origin counterpart such as rhabdomyosarcomas occurring in areas lacking skeletal muscle. Finally in vicarious evidence of sarcomas originating from pluripotential or partly committed stem cells, sarcomas frequently do not arise from their intuitive benign precursor unlike colonic carcinomas arising from colonic polyps. Welldifferentiated liposarcomas arise most commonly from deep soft tissues whereas lipomas usually occur in subcutaneous fat. Similarly uterine leiomyosarcomas do not arise from preceding uterine fibroids, monoclonal tumors derived from myometrium [62].

\section{FOXM1 AND EMBRYONIC PATHWAYS}

Enhanced FOXM1 stability may arise from altered rate of FOXM1 production or degradation. Embryonic/ stem cell pathways predominate in determining the cellular stoichiometry of FOXM1 levels. Wnt (effected by $\beta$-catenin) decreases the rate of FOXM1 degradation. Hedgehog signaling (effected by Gli) and Hippo signaling (effected by the YAP co-activator) upregulate FOXM1[63]. Hypoxia inducible factor-1 $\alpha$, also upregulates FOXM1. Tumors in which FOXM1 is implicated have a temporal incidence consistent with this biology, such as anaplastic large cell lymphoma being the most common childhood form of nonHodgkin's lymphoma. FOXM1 protein is stabilized in anaplastic large cell lymphoma by direct interaction with nucleophosmin (NPM). NPM is a fusion partner with ALK in $50 \%-75 \%$ of cases of anaplastic large cell lymphoma [64].

Frequently the interaction of FOXM1 with embryonal/stem cell preferential pathways is context dependent with variation occurring in different types of tumors. For example in endothelial cells FOXM1 has an unanticipated tumor suppressive function as it limits canonical Wnt signaling in lung epithelial cells [65]. Contrastingly, in gliomas FOXM1 promotes nuclear localization of $\beta$-catenin and expression of Wnt signaling pathway target genes [66]. Synovial sarcoma has a characteristic SYT-SSX translocation that increases Wnt pathway activity thereby imposing a differentiation block. Constitutive Wnt signaling activated by SYT-SSX2 has been identified in a SYT-SSX2 translocated mouse model [60]. Genetic loss of $\beta$-catenin prevents synovial sarcoma formation.

FOXM1 is a direct transcription target of hedgehog signaling [67]. Aberrant hedgehog signaling occurs in basal cell carcinoma, medulloblastoma, and chondrosarcoma. In embryonal and fusion gene negative alveolar rhabdomyosarcoma activation of hedgehog signaling is associated with a poor prognosis [68]. Hedgehog signaling is also activated in desmoid tumors, a locally recurrent, non-metastasizing fibromatosis more common in familial adenomatous polyposis coli or Gardner's syndrome with strong nuclear accumulation of $\beta$-catenin. Enchondromas, which are benign cartilaginous tumors, have constitutively active Hedgehog signaling which blocks the normal differentiation of chondrocytes and promotes cellular proliferation. $1 \%-2 \%$ of cases of solitary enchondromas transform to chondrosarcoma, with $15 \%-30 \%$ of enchondromatosis such as Ollier disease or Mafucci's syndromes transforming to chondrosarcoma. Indian Hedgehog, one of the 3 hedgehog orthologous, is constitutively activated in primary central chondrosarcoma $[69,70]$.

The Hippo pathway is a kinase cascade that negatively regulates the transcriptional co-activator YAP, causing its' cytoplasmic retention, proteasomal destruction and nuclear exclusion. The upstream coresignaling axis of this pathway comprises the serine/ threonine kinases MST1/2 followed by LATS1/2 [71]. Using information from The Cancer Genome Atlas Project, aberrant Hippo pathway signaling has been causally implicated in greater than $25 \%$ of sarcomas [31]. These comprise the histological subtypes fibrosarcoma, undifferentiated pleomorphic sarcoma, and liposarcoma. In this circumstance FOXM1 interacts directly with YAP (a transcription co-activator) via TEAD1 (the coassociating transcription factor) to alter regulation of pro-proliferative transcriptional targets. Inhibiting YAP decreases proliferation of sarcoma cells. YAP upregulates FOXM1. In murine models FOXM1 is required for sarcomagenesis. In a specific tumor type, dysregulation of Hippo signaling arises from a translocation fusion oncogene involving another FOX family member, FOXO1 in rhabdomyosarcoma. Rhabdomyosarcoma is the most common pediatric soft tissue sarcoma, accounting for $3 \%$ to $4 \%$ of all childhood cancers. Rhabdomyosarcoma can be either of alveolar or embryonal subtype. Alveolar rhabdomyosarcoma usually has a PAX3-FOXO1 translocation. This translocation oncogene permits bypass of cellular senescence by upregulating RASSF4, a Rasassociated domain family member. Enhanced RASSF4 expression inhibits MST1 to cause cell cycle progression and tumorigenesis [72]. YAP is upregulated in this circumstance.

\section{FOXM1 DIRECTED THERAPEUTICS}

There are numerous promising preclinical and therapeutic strategies, applying mono- or combinatorial approaches, to target the oncogenic transcription factor FOXM1. These include RNA interference, thiazole 
antibiotics and proteasome inhibitors, the appreciation that standard anticancer drugs such as cisplatin suppress FOXM1, and targeting FOXM1 interaction with other proteins using a synthetic ARF related peptide or nucleophosmin. In a selected specific, targeting FOXM1 retards p53-null sarcoma and lymphoma [41].

Preclinical studies that ablate FOXM1 by RNA interference have demonstrated decreased cellular proliferation in numerous types of cancer including osteosarcoma [29]. Knockdown of FOXM1 causes increased cellular senescence of mAKT1 expressing osteosarcoma cells and deletion of FOXM1 can diminish cancer cell invasion, migration and angiogenesis [73]. The thiazole antibiotics, siomycin A and thiostrepton act as proteosomal inhibitors and inhibit FOXM1 transcriptional activity $[74,75]$. Furthermore as FOXM1 has a positive auto regulation loop they also inhibit FOXM1 mRNA and protein expression [76]. In Ewing sarcoma thiostrepton has a dual inhibitory effect on FOXM1 and EWS/FLI mRNA expression [77]. Thiostrepton delays Ewing sarcoma xenograft growth in mice. FOXM1 is also a targeted for other proteasome inhibitors [74]. Bortezomib a proteasomal inhibitor already in clinical use has therapeutic efficacy previously attributed to suppression of NF- $\kappa$ B. Suppression of FOXM1 by proteasomal inhibition is an emerging alternative or complementary explanation [78]. In osteosarcoma and other cancer types a combination of bortezomib with thiostrepton induces profound apoptosis [79]. Several other anticancer drugs are also reported to repress FOXM1 including cisplatin, genistin (a chemo- preventive natural isoflavonoid in soya beans) and docetaxel [48].

The interaction of FOXM1 with other proteins can also be targeted. p19 $19^{\mathrm{ARF}}$ is one of the two gene transcripts and tumor suppressor products of the CDKN2A locus. It is a negative regulator of FOXM1. Amino acid residues 2644 of p19ARF bind to the c-terminal of the transcriptional activator domain of FOXM1 to cause inhibition of FOXM1 transcriptional activity by sequestering FOXM1 to the nucleolus. A synthetic ARF peptide comprising (D-Arg) ${ }_{9}$-p19 ${ }^{\text {ARF } 26-44 ~ h a s ~ b e e n ~ c r e a t e d . ~ T h i s ~ c o m p r i s e s ~}$ nine D-Arg residues to allow cell penetration, and amino acid residues 26-44 of the ARF protein.

This ARF peptide induces apoptosis, decreases cellular proliferation and prevents pulmonary metastasis in hepatocellular carcinoma [80, 81]. In preclinical experiments this synthetic peptide suppressed FOXM1 induced colony growth of U2OS osteosarcoma cells and suppressed FOXM1 transcriptional activity [82, 83]. Nucleophosmin (a protein associated with the nucleolus) directly interacts with FOXM1 and alters its localization and levels in cancer cells [R14]. Both nucleophosmin and FOXM1 tend to co-localize in the nucleolus of cancer cells and RNAi- mediated nucleophosmin knockdown in cancer cells decreases levels of FOXM1. Small molecules, which interfere with the nucleophosmin-FOXM1 interaction, decrease levels of FOXM1 and may be effective in treating cancer.

Targeting transcription factors with small molecules is an important emerging field. BET (bromodomain and extra terminal domain) proteins are epigenetic reader proteins that cause highly transcription factor specific repression of gene expression [84].

The family of BET proteins comprising BRD2, BRD3, BRD4 and BRDT, contain 2 conserved tandem bromodomains, which have an epigenetic reading function recognizing acetylated lysine residues on histone tails [85]. I-BET and JQ1 are small molecule BET inhibitors, which mimic the acetyl moiety, block the acetyl lysine binding pocket of the bomodomains, and displace the BET proteins from chromatin $[86,87]$. In many cancers the efficacy of BET inhibitors has been attributed to down regulation of the super enhancer dependent MYC transcript. However in contrast JQ1 markedly disrupts FOXM1 in sensitive ovarian cancer cells [88]. JQ1 is also efficacies in cell line and patient derived ovarian cancer xenograft models. Therefore epigenetic targeting of FOXM1 with small molecules is a potential therapeutic approach. JQ1 induces antiproliferative activity and apoptosis in osteosarcoma cells by suppressing the transcription factor FOSL1 [89]. This is mediated by displacing BRD4 from its locus. A synergistic increase in osteosarcoma cell sensitivity was observed with combined treatment of JQ1 with CDK inhibitors. The role of FOXM1 in this context requires future research.

FOXM1 can mediate drug sensitivity or resistance, and in breast cancer FOXM1 overexpression confers resistance to trastuzumab and paclitaxel. Stathmin a tubulin destabilizing protein that confers resistance to paclitaxel is a direct transcription target of FOXM1. In contra-distinction paclitaxel mediates it's anticancer activity by stabilizing the microtubule assembly. Attenuation of FOXM1 expression by RNA interference or alternative reading frame derived peptide inhibitors increased the therapeutic sensitivity of breast cancer to paclitaxel [90]. In pediatric B-acute lymphoblastic leukemia FOXM1 is overexpressed and in cell lines inhibition of FOXM1 with thiostrepton enhanced cytotoxicity to commonly used chemotherapeutic agents (dexamethasone, asparaginase, daunorubicin, vincristine and Ara-C) [91].

FOXM1 is overexpressed in many types of cancer and is usually absent in terminally differentiated cells. This differential expression renders it an attractive therapeutic target. It is likely that sarcoma subtypes with a high differentiation score are more likely to be sensitive to therapeutic inhibition of FOXM1. The commonly used three tiered FNCLCC (Fédération Nationale des Centres de Lutte Contre le Cancer) grading system of tumor differentiation, mitotic count, tumor necrosis and histologic grade are used as criteria to categorize sarcomas. The highest tumor differentiation score of 3 is 
assigned to the following sarcoma subtypes: round cell liposarcoma, pleomorphic liposarcoma, dedifferentiated liposarcoma, undifferentiated pleomorphic sarcoma, undifferentiated spindle cell sarcoma, poorly differentiated/pleomorphic/epithelioid leiomyosarcoma, biphasic/monophasic synovial sarcoma, pleomorphic rhabdomyosarcoma, mesenchymal chondrosarcoma, extraskeletal osteosarcoma, Ewing sarcoma/PNET, malignant rhabdoid tumors and some MFH variants. It is noteworthy that several sarcoma subtypes are exempted as grading of them is not recommended [92].

Nutlins are small molecule imidazoline compounds that selectively and potently inhibit MDM2 thereby stabilizing and activating p53 [93]. MDM2 is an E3 ligase, which binds p53, blocks the p53 transactivation domain and causes p53 degradation [94]. Nutlins suppress levels of FoxM1 in cell line studies. Inhibition of MDM2 by nutlins in liposarcoma activates the p53 pathway with decreased cellular proliferation in MDM2-amplified liposarcoma [95]. In one proof of principle study of 20 patients with well differentiated or dedifferentiated liposarcoma (18 TP53 wild-type, 2 TP53 missense mutant) treated with the nutlin RG7112, one patient had a complete response with fourteen patients experiencing stable disease. In a separate study involving melanoma, AMG 232 a small molecule that inhibits the MDM2-p53 interaction was combined with radiation treatment in cell lines and xenograft models [96]. Treatment with radiation or AMG 232 alone caused minor reductions in FOXM1 levels, whereas the combination treatment caused significant reductions in FOXM1 levels. This was detected by immunohistochemistry of treated A375 melanoma cells.

CDK4 is amplified in over $90 \%$ of welldifferentiated and de-differentiated liposarcomas. Palbociclib (a CDK4/6 inhibitor) is associated with a favorable progression free rate in RB expressing, CDK4 amplified well-differentiated and de-differentiated liposarcomas that experienced prior progression on systemic treatment [97]. FOXM1 is elevated in dedifferentiated liposarcoma and as FOXM1 is activated by progressive phosphorylation the relationship between palbociclib, CDK4 and FOXM1 in liposarcoma merits future research [31].

\section{CONCLUSIONS}

The central role of FOXM1 in the cell cycle, and it's recurring theme of importance in stem cell preferentially activated developmental pathways suggests that targeting FOXM1 is a potential advance in treating sarcoma. This review infers that certain sarcoma subtypes such as Ewing sarcoma and osteosarcoma may be particularly 'druggable' using FOXM1 inhibition. The accruing preclinical evidence presents a substantiate rationale for clinical trials in the future.

\section{CONFLICTS OF INTEREST}

The authors disclose no conflict of interest

\section{REFERENCES}

1. Laoukili J, Stahl M and Medema RH. FoxM1: at the crossroads of ageing and cancer. Biochimica et biophysica acta. 2007; 1775:92-102.

2. Halasi M and Gartel AL. FOX(M1) news - it is cancer. Molecular cancer therapeutics. 2013; 12:245-254.

3. Halasi $\mathrm{M}$ and Gartel AL. A novel mode of FoxM1 regulation: positive auto-regulatory loop. Cell cycle (Georgetown, Tex). 2009; 8:1966-1967.

4. Millour J, de Olano N, Horimoto Y, Monteiro LJ, Langer JK, Aligue R, Hajji N and Lam EW. ATM and p53 regulate FOXM1 expression via E2F in breast cancer epirubicin treatment and resistance. Molecular cancer therapeutics. 2011; 10:1046-1058.

5. Barsotti AM and Prives C. Pro-proliferative FoxM1 is a target of p53-mediated repression. Oncogene. 2009; 28:4295-4305

6. Wang IC, Snyder J, Zhang Y, Lander J, Nakafuku Y, Lin J, Chen G, Kalin TV, Whitsett JA and Kalinichenko VV. Foxm1 mediates cross talk between Kras/mitogenactivated protein kinase and canonical Wnt pathways during development of respiratory epithelium. Molecular and cellular biology. 2012; 32:3838-3850.

7. Anders L, Ke N, Hydbring P, Choi YJ, Widlund HR, Chick JM, Zhai H, Vidal M, Gygi SP, Braun P and Sicinski P. A systematic screen for CDK4/6 substrates links FOXM1 phosphorylation to senescence suppression in cancer cells. Cancer cell. 2011; 20:620-634.

8. Major ML, Lepe R and Costa RH. Forkhead box M1B transcriptional activity requires binding of Cdk-cyclin complexes for phosphorylation-dependent recruitment of p300/CBP coactivators. Molecular and cellular biology. 2004; 24:2649-2661.

9. Ma RY, Tong TH, Cheung AM, Tsang AC, Leung WY and Yao KM. Raf/MEK/MAPK signaling stimulates the nuclear translocation and transactivating activity of FOXM1c. Journal of cell science. 2005; 118:795-806.

10. Choi YJ and Anders L. Signaling through cyclin D-dependent kinases. Oncogene. 2014; 33:1890-1903.

11. Finn RS, Crown JP, Lang I, Boer K, Bondarenko IM, Kulyk SO, Ettl J, Patel R, Pinter T, Schmidt M, Shparyk Y, Thummala AR, Voytko NL, Fowst C, Huang X, Kim ST, et al. The cyclin-dependent kinase 4/6 inhibitor palbociclib in combination with letrozole versus letrozole alone as first-line treatment of oestrogen receptor-positive, HER2negative, advanced breast cancer (PALOMA-1/TRIO-18): a randomised phase 2 study. The Lancet Oncology. 2015; 16:25-35.

12. Belchis DA, Meece CA, Benko FA, Rogan PK, 
Williams RA and Gocke CD. Loss of heterozygosity and microsatellite instability at the retinoblastoma locus in osteosarcomas. Diagnostic molecular pathology : the American journal of surgical pathology, part B. 1996; 5:214-219.

13. Feugeas O, Guriec N, Babin-Boilletot A, Marcellin L, Simon P, Babin S, Thyss A, Hofman P, Terrier P, Kalifa C, Brunat-Mentigny M, Patricot LM and Oberling F. Loss of heterozygosity of the RB gene is a poor prognostic factor in patients with osteosarcoma. Journal of clinical oncology. 1996; 14:467-472.

14. Wadayama B, Toguchida J, Shimizu T, Ishizaki K, Sasaki MS, Kotoura Y and Yamamuro T. Mutation spectrum of the retinoblastoma gene in osteosarcomas. Cancer research. 1994; 54:3042-3048.

15. Wei G, Lonardo F, Ueda T, Kim T, Huvos AG, Healey JH and Ladanyi M. CDK4 gene amplification in osteosarcoma: reciprocal relationship with INK4A gene alterations and mapping of $12 \mathrm{q} 13$ amplicons. International journal of cancer Journal international du cancer. 1999; 80:199-204.

16. Marees T, Moll AC, Imhof SM, de Boer MR, Ringens $\mathrm{PJ}$ and van Leeuwen FE. Risk of second malignancies in survivors of retinoblastoma: more than 40 years of follow-up. Journal of the National Cancer Institute. 2008; 100:1771-1779.

17. MacCarthy A, Bayne AM, Brownbill PA, Bunch KJ, Diggens NL, Draper GJ, Hawkins MM, Jenkinson HC, Kingston JE, Stiller CA, Vincent TJ and Murphy MF. Second and subsequent tumours among 1927 retinoblastoma patients diagnosed in Britain 1951-2004. British journal of cancer. 2013; 108:2455-2463.

18. Sadasivam S and DeCaprio JA. The DREAM complex: master coordinator of cell cycle-dependent gene expression. Nature reviews Cancer. 2013; 13:585-595.

19. Zahm SH and Fraumeni JF, Jr. The epidemiology of soft tissue sarcoma. Seminars in oncology. 1997; 24:504-514.

20. Overholtzer M, Rao PH, Favis R, Lu XY, Elowitz MB, Barany F, Ladanyi M, Gorlick R and Levine AJ. The presence of $\mathrm{p} 53$ mutations in human osteosarcomas correlates with high levels of genomic instability. Proceedings of the National Academy of Sciences of the United States of America. 2003; 100:11547-11552.

21. Gokgoz N, Wunder JS, Mousses S, Eskandarian S, Bell RS and Andrulis IL. Comparison of p53 mutations in patients with localized osteosarcoma and metastatic osteosarcoma. Cancer. 2001; 92:2181-2189.

22. Parikh N, Hilsenbeck S, Creighton CJ, Dayaram $T$, Shuck R, Shinbrot E, Xi L, Gibbs RA, Wheeler DA and Donehower LA. Effects of TP53 mutational status on gene expression patterns across 10 human cancer types. The Journal of pathology. 2014; 232:522-533.

23. Mowla SN, Lam EW and Jat PS. Cellular senescence and aging: the role of B-MYB. Aging cell. 2014; 13:773-779.

24. Down CF, Millour J, Lam EW and Watson RJ. Binding of
FoxM1 to G2/M gene promoters is dependent upon B-Myb. Biochimica et biophysica acta. 2012; 1819:855-862.

25. Wonsey DR and Follettie MT. Loss of the forkhead transcription factor FoxM1 causes centrosome amplification and mitotic catastrophe. Cancer research. 2005; 65:51815189.

26. Leung TW, Lin SS, Tsang AC, Tong CS, Ching JC, Leung WY, Gimlich R, Wong GG and Yao KM. Over-expression of FoxM1 stimulates cyclin B1 expression. FEBS letters. 2001; 507:59-66.

27. Imai Y, Takahashi A, Hanyu A, Hori S, Sato S, Naka K, Hirao A, Ohtani N and Hara E. Crosstalk between the Rb pathway and AKT signaling forms a quiescence-senescence switch. Cell reports. 2014; 7:194-207.

28. Yu J, Deshmukh H, Payton JE, Dunham C, Scheithauer BW, Tihan T, Prayson RA, Guha A, Bridge JA, Ferner RE, Lindberg GM, Gutmann RJ, Emnett RJ, Salavaggione L, Gutmann DH, Nagarajan R, et al. Array-based comparative genomic hybridization identifies CDK4 and FOXM1 alterations as independent predictors of survival in malignant peripheral nerve sheath tumor. Clinical cancer research. 2011; 17:1924-1934.

29. Wang IC, Chen YJ, Hughes D, Petrovic V, Major ML, Park HJ, Tan Y, Ackerson T and Costa RH. Forkhead box M1 regulates the transcriptional network of genes essential for mitotic progression and genes encoding the SCF (Skp2Cks1) ubiquitin ligase. Molecular and cellular biology. 2005; 25:10875-10894.

30. Xie Z, Tan G, Ding M, Dong D, Chen T, Meng X, Huang $\mathrm{X}$ and Tan Y. Foxm1 transcription factor is required for maintenance of pluripotency of P19 embryonal carcinoma cells. Nucleic acids research. 2010; 38:8027-8038.

31. Eisinger-Mathason TS, Mucaj V, Biju KM, Nakazawa MS, Gohil M, Cash TP, Yoon SS, Skuli N, Park KM, Gerecht $\mathrm{S}$ and Simon MC. Deregulation of the Hippo pathway in soft-tissue sarcoma promotes FOXM1 expression and tumorigenesis. Proceedings of the National Academy of Sciences of the United States of America. 2015; 112:E34023411.

32. Korver W, Roose J, Heinen K, Weghuis DO, de Bruijn D, van Kessel AG and Clevers H. The human TRIDENT/HFH11/FKHL16 gene: structure, localization, and promoter characterization. Genomics. 1997; 46:435-442.

33. Uddin S, Hussain AR, Ahmed M, Siddiqui K, Al-Dayel F, Bavi P and Al-Kuraya KS. Overexpression of FoxM1 offers a promising therapeutic target in diffuse large B-cell lymphoma. Haematologica. 2012; 97:1092-1100.

34. Dei Tos AP and Dal Cin P. The role of cytogenetics in the classification of soft tissue tumours. Virchows Archiv : an international journal of pathology. 1997; 431:83-94.

35. Mantripragada KK, Spurlock G, Kluwe L, Chuzhanova N, Ferner RE, Frayling IM, Dumanski JP, Guha A, Mautner $\mathrm{V}$ and Upadhyaya M. High-resolution DNA copy number profiling of malignant peripheral nerve sheath tumors 
using targeted microarray-based comparative genomic hybridization. Clinical cancer research. 2008; 14:10151024.

36. Kresse SH, Skarn M, Ohnstad HO, Namlos HM, Bjerkehagen B, Myklebost O and Meza-Zepeda LA. DNA copy number changes in high-grade malignant peripheral nerve sheath tumors by array $\mathrm{CGH}$. Molecular cancer. 2008; 7:48.

37. Pandit B, Halasi M and Gartel AL. p53 negatively regulates expression of FoxM1. Cell cycle (Georgetown, Tex). 2009; 8:3425-3427.

38. Meek DW. Tumour suppression by p53: a role for the DNA damage response? Nature reviews Cancer. 2009; 9:714-723.

39. Donehower LA, Harvey M, Slagle BL, McArthur MJ, Montgomery CA, Jr., Butel JS and Bradley A. Mice deficient for p53 are developmentally normal but susceptible to spontaneous tumours. Nature. 1992; 356:215221.

40. Li FP, Fraumeni JF, Jr., Mulvihill JJ, Blattner WA, Dreyfus MG, Tucker MA and Miller RW. A cancer family syndrome in twenty-four kindreds. Cancer research. 1988; 48:53585362 .

41. Wang Z, Zheng Y, Park HJ, Li J, Carr JR, Chen YJ, Kiefer MM, Kopanja D, Bagchi S, Tyner AL and Raychaudhuri P. Targeting FoxM1 effectively retards p53-null lymphoma and sarcoma. Molecular cancer therapeutics. 2013; 12:759767.

42. Wierstra I. The transcription factor FOXM1c binds to and transactivates the promoter of the tumor suppressor gene E-cadherin. Cell cycle (Georgetown, Tex). 2011; 10:760766.

43. Kong FF, Qu ZQ, Yuan HH, Wang JY, Zhao M, Guo YH, Shi J, Gong XD, Zhu YL, Liu F, Zhang WY and Jiang B. Overexpression of FOXM1 is associated with EMT and is a predictor of poor prognosis in non-small cell lung cancer. Oncology reports. 2014; 31:2660-2668.

44. Hanks S, Coleman K, Reid S, Plaja A, Firth H, Fitzpatrick D, Kidd A, Mehes K, Nash R, Robin N, Shannon N, Tolmie J, Swansbury J, Irrthum A, Douglas J and Rahman N. Constitutional aneuploidy and cancer predisposition caused by biallelic mutations in BUB1B. Nature genetics. 2004; 36:1159-1161.

45. Wan X, Yeung C, Kim SY, Dolan JG, Ngo VN, Burkett S, Khan J, Staudt LM and Helman LJ. Identification of FoxM1/Bub1b signaling pathway as a required component for growth and survival of rhabdomyosarcoma. Cancer research. 2012; 72:5889-5899.

46. Matsuura S, Matsumoto $\mathrm{Y}$, Morishima $\mathrm{K}$, Izumi $\mathrm{H}$, Matsumoto H, Ito E, Tsutsui K, Kobayashi J, Tauchi H, Kajiwara Y, Hama S, Kurisu K, Tahara H, Oshimura M, Komatsu K, Ikeuchi T, et al. Monoallelic BUB1B mutations and defective mitotic-spindle checkpoint in seven families with premature chromatid separation (PCS) syndrome. American journal of medical genetics Part A. 2006;
140:358-367.

47. Ognjanovic S, Linabery AM, Charbonneau B and Ross JA. Trends in childhood rhabdomyosarcoma incidence and survival in the United States, 1975-2005. Cancer. 2009; 115:4218-4226.

48. Wang Z, Park HJ, Carr JR, Chen YJ, Zheng Y, Li J, Tyner AL, Costa RH, Bagchi S and Raychaudhuri P. FoxM1 in tumorigenicity of the neuroblastoma cells and renewal of the neural progenitors. Cancer research. 2011; 71:42924302 .

49. Christensen L, Joo J, Lee S, Wai D, Triche TJ and May WA. FOXM1 is an oncogenic mediator in Ewing Sarcoma. PLoS One. 2013; 8:e54556.

50. Riggi N, Suva ML, De Vito C, Provero P, Stehle JC, Baumer K, Cironi L, Janiszewska M, Petricevic T, Suva D, Tercier S, Joseph JM, Guillou L and Stamenkovic I. EWS-FLI-1 modulates miRNA145 and SOX2 expression to initiate mesenchymal stem cell reprogramming toward Ewing sarcoma cancer stem cells. Genes \& development. 2010; 24:916-932.

51. Ren C, Ren T, Yang K, Wang S, Bao X, Zhang F and Guo W. Inhibition of SOX2 induces cell apoptosis and G1/S arrest in Ewing's sarcoma through the PI3K/Akt pathway. Journal of experimental \& clinical cancer research : CR. 2016; 35:44.

52. Suva ML, Riggi N, Stehle JC, Baumer K, Tercier S, Joseph JM, Suva D, Clement V, Provero P, Cironi L, Osterheld MC, Guillou L and Stamenkovic I. Identification of cancer stem cells in Ewing's sarcoma. Cancer research. 2009; 69:1776-1781.

53. Trucco M and Loeb D. Sarcoma stem cells: do we know what we are looking for? Sarcoma. 2012; 2012:291705.

54. Jiang X, Gwye Y, Russell D, Cao C, Douglas D, Hung L, Kovar H, Triche TJ and Lawlor ER. CD133 expression in chemo-resistant Ewing sarcoma cells. BMC cancer. 2010; 10:116.

55. Salerno M, Avnet S, Bonuccelli G, Eramo A, De Maria R, Gambarotti M, Gamberi G and Baldini N. Sphereforming cell subsets with cancer stem cell properties in human musculoskeletal sarcomas. International journal of oncology. 2013; 43:95-102.

56. Svejstrup JQ. Synovial sarcoma mechanisms: a series of unfortunate events. Cell. 2013; 153:11-12.

57. Kadoch $\mathrm{C}$ and Crabtree GR. Reversible disruption of $\mathrm{mSWI} / \mathrm{SNF}$ (BAF) complexes by the SS18-SSX oncogenic fusion in synovial sarcoma. Cell. 2013; 153:71-85.

58. Basu-Roy U, Bayin NS, Rattanakorn K, Han E, Placantonakis DG, Mansukhani A and Basilico C. Sox2 antagonizes the Hippo pathway to maintain stemness in cancer cells. Nature communications. 2015; 6:6411.

59. Basu-Roy U, Seo E, Ramanathapuram L, Rapp TB, Perry JA, Orkin SH, Mansukhani A and Basilico C. Sox2 maintains self renewal of tumor-initiating cells in osteosarcomas. Oncogene. 2012; 31:2270-2282. 
60. Barham W, Frump AL, Sherrill TP, Garcia CB, SaitoDiaz K, VanSaun MN, Fingleton B, Gleaves L, Orton D, Capecchi MR, Blackwell TS, Lee E, Yull F and Eid JE. Targeting the Wnt pathway in synovial sarcoma models. Cancer discovery. 2013; 3:1286-1301.

61. Chen Y, Meng L, Yu Q, Dong D, Tan G, Huang X and Tan Y. The miR-134 attenuates the expression of transcription factor FOXM1 during pluripotent NT2/D1 embryonal carcinoma cell differentiation. Experimental cell research. 2015; 330:442-450.

62. Quade BJ, Wang TY, Sornberger K, Dal Cin P, Mutter GL and Morton CC. Molecular pathogenesis of uterine smooth muscle tumors from transcriptional profiling. Genes, chromosomes \& cancer. 2004; 40:97-108.

63. Katoh M, Igarashi M, Fukuda H, Nakagama $\mathrm{H}$ and Katoh M. Cancer genetics and genomics of human FOX family genes. Cancer letters. 2013; 328:198-206.

64. Morris SW, Kirstein MN, Valentine MB, Dittmer KG, Shapiro DN, Saltman DL and Look AT. Fusion of a kinase gene, ALK, to a nucleolar protein gene, NPM, in nonHodgkin's lymphoma. Science (New York, NY). 1994; 263:1281-1284.

65. Wierstra I. FOXM1 (Forkhead box M1) in tumorigenesis: overexpression in human cancer, implication in tumorigenesis, oncogenic functions, tumor-suppressive properties, and target of anticancer therapy. Advances in cancer research. 2013; 119:191-419.

66. Zhang N, Wei P, Gong A, Chiu WT, Lee HT, Colman H, Huang H, Xue J, Liu M, Wang Y, Sawaya R, Xie K, Yung WK, Medema RH, He X and Huang S. FoxM1 promotes beta-catenin nuclear localization and controls Wnt targetgene expression and glioma tumorigenesis. Cancer cell. 2011; 20:427-442.

67. Katoh Y and Katoh M. Hedgehog signaling pathway and gastric cancer. Cancer biology \& therapy. 2005; 4:10501054.

68. Zibat A, Missiaglia E, Rosenberger A, Pritchard-Jones K, Shipley J, Hahn H and Fulda S. Activation of the hedgehog pathway confers a poor prognosis in embryonal and fusion gene-negative alveolar rhabdomyosarcoma. Oncogene. 2010; 29:6323-6330.

69. Tiet TD, Hopyan S, Nadesan P, Gokgoz N, Poon R, Lin AC, Yan T, Andrulis IL, Alman BA and Wunder JS. Constitutive hedgehog signaling in chondrosarcoma upregulates tumor cell proliferation. The American journal of pathology. 2006; 168:321-330.

70. Schrage YM, Hameetman L, Szuhai K, Cleton-Jansen AM, Taminiau AH, Hogendoorn PC and Bovee JV. Aberrant heparan sulfate proteoglycan localization, despite normal exostosin, in central chondrosarcoma. The American journal of pathology. 2009; 174:979-988.

71. Kelleher FC and O'Sullivan H. Oxford and the Savannah: can the hippo provide an explanation for Peto's paradox? Clinical cancer research. 2014; 20:557-564.
72. Crose LE, Galindo KA, Kephart JG, Chen C, Fitamant J, Bardeesy N, Bentley RC, Galindo RL, Chi JT and Linardic CM. Alveolar rhabdomyosarcoma-associated PAX3-FOXO1 promotes tumorigenesis via Hippo pathway suppression. The Journal of clinical investigation. 2014; 124:285-296.

73. Park HJ, Carr JR, Wang Z, Nogueira V, Hay N, Tyner AL, Lau LF, Costa RH and Raychaudhuri P. FoxM1, a critical regulator of oxidative stress during oncogenesis. The EMBO journal. 2009; 28:2908-2918.

74. Bhat UG, Halasi M and Gartel AL. FoxM1 is a general target for proteasome inhibitors. PLoS One. 2009; 4:e6593.

75. Bhat UG, Halasi M and Gartel AL. Thiazole antibiotics target FoxM1 and induce apoptosis in human cancer cells. PLoS One. 2009; 4:e5592.

76. Gartel AL. FoxM1 inhibitors as potential anticancer drugs. Expert opinion on therapeutic targets. 2008; 12:663-665.

77. Sengupta A, Rahman M, Mateo-Lozano S, Tirado OM and Notario V. The dual inhibitory effect of thiostrepton on FoxM1 and EWS/FLI1 provides a novel therapeutic option for Ewing's sarcoma. International journal of oncology. 2013; 43:803-812.

78. Halasi $\mathrm{M}$ and Gartel AL. Targeting FOXM1 in cancer. Biochemical pharmacology. 2013; 85:644-652.

79. Pandit B and Gartel AL. Thiazole antibiotic thiostrepton synergize with bortezomib to induce apoptosis in cancer cells. PLoS One. 2011; 6:e17110.

80. Park HJ, Gusarova G, Wang Z, Carr JR, Li J, Kim KH, Qiu J, Park YD, Williamson PR, Hay N, Tyner AL, Lau LF, Costa RH and Raychaudhuri P. Deregulation of FoxM1b leads to tumour metastasis. EMBO molecular medicine. 2011; 3:21-34.

81. Gusarova GA, Wang IC, Major ML, Kalinichenko VV, Ackerson T, Petrovic V and Costa RH. A cell-penetrating ARF peptide inhibitor of FoxM1 in mouse hepatocellular carcinoma treatment. The Journal of clinical investigation. 2007; 117:99-111.

82. Kalinichenko VV, Major ML, Wang X, Petrovic V, Kuechle J, Yoder HM, Dennewitz MB, Shin B, Datta A, Raychaudhuri P and Costa RH. Foxm1b transcription factor is essential for development of hepatocellular carcinomas and is negatively regulated by the p19ARF tumor suppressor. Genes \& development. 2004; 18:830-850.

83. Costa RH, Kalinichenko VV, Major ML and Raychaudhuri P. New and unexpected: forkhead meets ARF. Current opinion in genetics \& development. 2005; 15:42-48.

84. Hagenbuchner $\mathrm{J}$ and Ausserlechner MJ. Targeting transcription factors by small compounds-Current strategies and future implications. Biochemical pharmacology. 2015.

85. Filippakopoulos $\mathrm{P}$ and Knapp S. Targeting bromodomains: epigenetic readers of lysine acetylation. Nature reviews Drug discovery. 2014; 13:337-356.

86. Filippakopoulos P, Qi J, Picaud S, Shen Y, Smith WB, Fedorov O, Morse EM, Keates T, Hickman TT, Felletar I, 
Philpott M, Munro S, McKeown MR, Wang Y, Christie AL, West N, et al. Selective inhibition of BET bromodomains. Nature. 2010; 468:1067-1073.

87. Dawson MA, Prinjha RK, Dittmann A, Giotopoulos G, Bantscheff M, Chan WI, Robson SC, Chung CW, Hopf C, Savitski MM, Huthmacher C, Gudgin E, Lugo D, Beinke $\mathrm{S}$, Chapman TD, Roberts EJ, et al. Inhibition of BET recruitment to chromatin as an effective treatment for MLLfusion leukaemia. Nature. 2011; 478:529-533.

88. Zhang Z, Ma P, Jing Y, Yan Y, Cai MC, Zhang M, Zhang S, Peng H, Ji ZL, Di W, Gu Z, Gao WQ and Zhuang G. BET Bromodomain Inhibition as a Therapeutic Strategy in Ovarian Cancer by Downregulating FoxM1. Theranostics. 2016; 6:219-230.

89. Baker EK, Taylor S, Gupte A, Sharp PP, Walia M, Walsh NC, Zannettino AC, Chalk AM, Burns CJ and Walkley CR. BET inhibitors induce apoptosis through a MYC independent mechanism and synergise with $\mathrm{CDK}$ inhibitors to kill osteosarcoma cells. Scientific reports. 2015; 5:10120.

90. Carr JR, Park HJ, Wang Z, Kiefer MM and Raychaudhuri P. FoxM1 mediates resistance to herceptin and paclitaxel. Cancer research. 2010; 70:5054-5063.

91. Consolaro F, Basso G, Ghaem-Magami S, Lam EW and Viola G. FOXM1 is overexpressed in B-acute lymphoblastic leukemia (B-ALL) and its inhibition sensitizes B-ALL cells to chemotherapeutic drugs. International journal of oncology. 2015; 47:1230-1240.

92. Guillou L, Coindre JM, Bonichon F, Nguyen BB, Terrier P, Collin F, Vilain MO, Mandard AM, Le Doussal V, Leroux A, Jacquemier J, Duplay H, Sastre-Garau X and Costa J. Comparative study of the National Cancer Institute and French Federation of Cancer Centers Sarcoma Group grading systems in a population of 410 adult patients with soft tissue sarcoma. Journal of clinical oncology. 1997; 15:350-362.
93. Manfredi JJ. The Mdm2-p53 relationship evolves: Mdm2 swings both ways as an oncogene and a tumor suppressor. Genes \& development. 2010; 24:1580-1589.

94. Kussie PH, Gorina S, Marechal V, Elenbaas B, Moreau J, Levine AJ and Pavletich NP. Structure of the MDM2 oncoprotein bound to the p53 tumor suppressor transactivation domain. Science (New York, NY). 1996; 274:948-953.

95. Ray-Coquard I, Blay JY, Italiano A, Le Cesne A, Penel N, Zhi J, Heil F, Rueger R, Graves B, Ding M, Geho D, Middleton SA, Vassilev LT, Nichols GL and Bui BN. Effect of the MDM2 antagonist RG7112 on the P53 pathway in patients with MDM2-amplified, well-differentiated or dedifferentiated liposarcoma: an exploratory proof-ofmechanism study. The Lancet Oncology. 2012; 13:11331140.

96. Werner LR, Huang S, Francis DM, Armstrong EA, Ma F, Li C, Iyer G, Canon J and Harari PM. Small Molecule Inhibition of MDM2-p53 Interaction Augments Radiation Response in Human Tumors. Molecular cancer therapeutics. 2015; 14:1994-2003.

97. Dickson MA, Tap WD, Keohan ML, D'Angelo SP, Gounder MM, Antonescu CR, Landa J, Qin LX, Rathbone DD, Condy MM, Ustoyev Y, Crago AM, Singer S and Schwartz GK. Phase II trial of the CDK4 inhibitor PD0332991 in patients with advanced CDK4-amplified well-differentiated or dedifferentiated liposarcoma. Journal of clinical oncology. 2013; 31:2024-2028. 